\title{
Role of interleukin-6 in ischemic stroke outcome
}

\author{
Hani Mohamed Amin Aref, Nagia Ali Fahmy, Salma Hamed Khalil, Mai Fathy Ahmed, Ahmed ElSadek ${ }^{*}$ and \\ Mohamed Osama Abdulghani
}

\begin{abstract}
Background: High levels of inflammatory markers such as C-reactive protein (CRP) and interleukin (IL)-6 are associated with worse outcome after ischemic stroke.

Objectives: To study whether the level of IL-6 following ischemic stroke is related to the severity of stroke, infarct volume, stroke subtype, and its impact on stroke outcome.

Materials and methods: A total of 90 patients of acute ischemic stroke were enrolled with calculation of infarct volume in MRI brain, and serum IL-6 samples were obtained within $24 \mathrm{~h}$ and also after 3 months from stroke onset. NIHSS and MRS were done at stroke onset and after 3 months.

Results: The mean value of IL-6 was higher among patients with small vessel occlusion $(8.47 \mathrm{pg} / \mathrm{ml})$ with a significant positive correlation between IL-6 and National Institute of Health Stroke Scale (NIHSS) and modified Rankin Scale (MRS) after 3 months. IL-6 was significantly higher in patients with recurrence with a cutoff IL-6 value above which recurrence is anticipated which is $7.75 \mathrm{pg} / \mathrm{ml}$.

Conclusion: Our study concluded that inflammation has an important role in the pathogenesis of acute ischemic stroke particularly small vessel occlusion and it provided evidence that IL-6 contributes to the determination of clinical outcome of acute ischemic stroke and prediction of recurrence.
\end{abstract}

Keywords: Interleukin-6, Ischemic stroke, Stroke outcome

\section{Introduction}

Inflammatory response plays an essential role in the pathophysiology of acute ischemic stroke (AIS) [1]. Plasma level of various inflammatory markers is noted to be higher in stroke patients compared to healthy populations [2]. On the basis of the pathophysiologic differences between different stroke subtypes, it is possible to hypothesize a different pattern of immuno-inflammatory activation in relation to those subtypes. A nonspecific systemic inflammatory response occurs after either ischemic or hemorrhagic stroke as a part of the process of brain damage. Elevated levels of inflammatory markers such as C-reactive protein (CRP) and interleukin-6 are associated with poor outcome after ischemic stroke. Interleukin- 6 can cause the release of prostaglandin $E_{2}$ in the brain, and prostaglandin $E_{2}$ activates the hypothalamus leading to an increase in body temperature.

* Correspondence: ahmedelsadek_79@yahoo.com

Ain Shams University, El Rehab, Cairo, Egypt
Different studies reported that fever is correlated with increased size of infarction and poor stroke outcome; thus, the early and prolonged elevation in interleukin-6 in the CSF and blood most likely correlates with increased risk of fever that increases tissue damage after ischemic stroke [3]. Significant increase of interleukin-6 levels have been reported in stroke patients shortly after the ischemic event. All major brain tissue cell types are capable of synthesizing interleukin-6 [4].

\section{Aim of this work}

This research aims to study whether the level of IL-6 following ischemic stroke is related to the severity of stroke, infarct volume, stroke subtype, and its impact on stroke outcome.

\section{Materials and methods}

The study subjects which included 90 patients of acute ischemic stroke within $24 \mathrm{~h}$ from stroke onset were 
recruited to this prospective cohort study from the stroke unit at Ain Shams University hospitals during the period from December 2014 to October 2015. All patients had detailed neurologic history, examination, and investigations to determine different stroke mechanisms and their risk factors. Neurological status of our subjects was assessed via the National Institute of Health Stroke Scale (NIHSS) and modified Rankin Scale (mRS) after which was done on admission and after 3 months for follow-up. We consider NIHSS more than 21 as poor prognosis and less than 5 as good prognosis. All patients had magnetic resonance imaging (MRI) of the brain (diffusion-weighted, flair, T1, T2, and T2* images) and magnetic resonance angiography (MRA) (Philips 1.5 Tesla, Germany). The infarct volume can be calculated (approximately) using the formula $A B C / 2$, where $A, B$, and $C$ are the longest 3 orthogonal distances of the lesion as measured [5]. According to infarction volume, small vessel disease was considered less than that of $508 \mathrm{~mm}^{3}$, while large vessel disease those exceeding $508 \mathrm{~mm}^{3}$ [6]. Serum IL-6 samples were collected in the first $24 \mathrm{~h}$ from stroke onset. Serum was collected and stored at $-70^{\circ} \mathrm{C}$ after centrifugation of the blood samples at $3000 \times g$ for $10 \mathrm{~min}$. For the quantitative detection of IL-6, the human IL-6 enzyme-linked immunosorbent assay (ELISA) kit which was provided by Assaypro LLC (number EI1006-1) was used and the instructions were done according to the provided catalog in the laboratory of Ain Shams University hospitals. Circulating IL- 6 can be found in the blood of normal individuals in the $1 \mathrm{pg} / \mathrm{ml}$ range $[7,8]$. Carotid duplex (General Electric Logic 5, USA), transthoracic echocardiography, and transesophageal echocardiography (if needed) (General Electric S5, USA) were done for all the studied patients.

\section{Inclusion criteria and subject selection}

A clinical presentation of the first ever acute ischemic cerebrovascular stroke, within $24 \mathrm{~h}$ from stroke onset (defined according to WHO definition as the presence of rapidly developing focal neurological signs or symptoms of vascular origin that persists for more than $1 \mathrm{~h}$ with evidence of acute infarction on diffusion-weighted imaging in MRI) [9].

\section{Exclusion criteria}

Patients were excluded from enrollment if age is less than 18 years, other determined etiology and undetermined etiology of stroke subtypes according to the Trial of ORG 10712 in Acute Stroke Treatment (TOAST) classification [10], presence of cancer, receiving thrombolytic therapy, and systemic disorders (inflammatory or infectious diseases, hematological diseases, rheumatologic diseases, severe renal or liver failure, patients who were under treatment with anti-inflammatory drugs, and immunosuppressive or immunomodulatory
Table 1 Description of compliance, recurrence and mortality among cases

\begin{tabular}{lll}
\hline & No & Yes \\
& $\%(N)$ & $\%(N)$ \\
\hline Compliance & $2.3 \%(2)$ & $97.7 \%(88)$ \\
Recurrence & $83.3 \%(75)$ & $16.7 \%(15)$ \\
Mortality & $88.9 \%(80)$ & $11.1 \%(10)$ \\
\hline
\end{tabular}

drugs for any medical disease in the last 6 months). Patients with fever of unknown etiology or with recent venous thromboembolism. Laboratory investigations were done for all patients to exclude those causes (complete blood picture, ESR, CRP, uric acid, renal function test, liver function test, collagen profile, urine analysis)

\section{Ethical consideration}

The study was approved by Ain Shams University Ethical Committee in December 2014. Written informed consent was obtained from the patients participating in the study, or their first-degree relatives after informing them about the study rationale and their right to withdraw from the study at any time without any consequences.

\section{Statistical analysis}

Statistical analysis was conducted using SPSS $\times 7$ (SPSS, IBM, Chicago, IL, USA). $P$ values were considered significant if $<0.05$. Descriptive statistics were used for patient demographics, Chi square test was used to compare normally distributed demographics, and likelihood ratio was used to compare abnormally distributed variables, in addition to recurrence, and cardiovascularrelated deaths, among the different stroke subgroups. $T$ test was used to compare means of NIHSS on admission and at 3 months, while Wilcoxon signed-rank test was used to compare the means of mRS at admission and at 3 months. Kruskal-Wallis test was used to compare mean values of IL-6 among different stroke subtypes. Kaplan-Meier survival curves were plotted to display time of death, and receiver operating characteristics (ROC) with area under the curve (AUC) analyses were used to detect IL- 6 value testing sensitivity and specificity in predicting recurrence.

Table 2 Comparing means of IL-6 values among different stroke subgroups

\begin{tabular}{lllll}
\hline & Number & $\begin{array}{l}\text { Mean } \\
(\mathrm{pg} / \mathrm{ml})\end{array}$ & $\begin{array}{l}\text { 95\% Confidence } \\
\text { interval }\end{array}$ & $P$ value \\
\hline Cardioembolic & 27 & 4.64 & $3.37-5.92$ & $0.005^{*}$ \\
LAA & 25 & 5.11 & $3.3-6.92$ & \\
SVO & 38 & 8.47 & $6.82-10.12$ & \\
Total & 90 & 6.39 & $5.41-7.37$ &
\end{tabular}

${ }^{*} P$ value is calculated using Kruskal-Wallis test; $0.005=$ significant 
Table 3 Correlation between IL-6 levels and both NIHSS and mRS levels on admission and after 3 months

\begin{tabular}{lllll}
\hline & & $r$ value & $P$ value & Significance \\
\hline IL-6 & 0.135 & $0.2^{*}$ & Non-statistically significant positive correlation \\
& mRS on admission & 0.123 & $0.24^{*}$ & Non-statistically significant positive correlation \\
NIHSS at 3 months & 0.28 & $0.009^{*}$ & Statistically significant positive correlation \\
mRS at 3 months & 0.42 & $<0.0001^{*}$ & Statistically significant positive correlation \\
\hline
\end{tabular}

*P value is calculated using Pearson's correlation

\section{Results}

Those 90 patients with acute ischemic cerebrovascular stroke included 48 males $(53.3 \%)$ and 42 females (46.7\%), and their age ranged from 28 to 88 years with the mean age of $60.18 \pm 12.19$ years.

\section{Risk factors}

Diabetes mellitus was the most prevalent risk factor in 59 patients $(65.6 \%)$, followed by hypertension in 56 patients $(62.2 \%)$, followed by dyslipidemia in 49 patients
(54.4\%), followed by smoking in 32 patients (35.6\%), followed by ischemic heart disease (ISHD) in 24 patients (26.7\%), and followed by AF in 19 patients (21.1\%).

\section{Stroke site}

Sixty-four ischemic strokes were located anteriorly, i.e., in the distribution of anterior cerebral artery or middle cerebral artery (MCA) (71.1\%) and 26 ischemic strokes were located posteriorly in the distribution of the vertebral, basilar, or posterior cerebral artery (28.9\%).

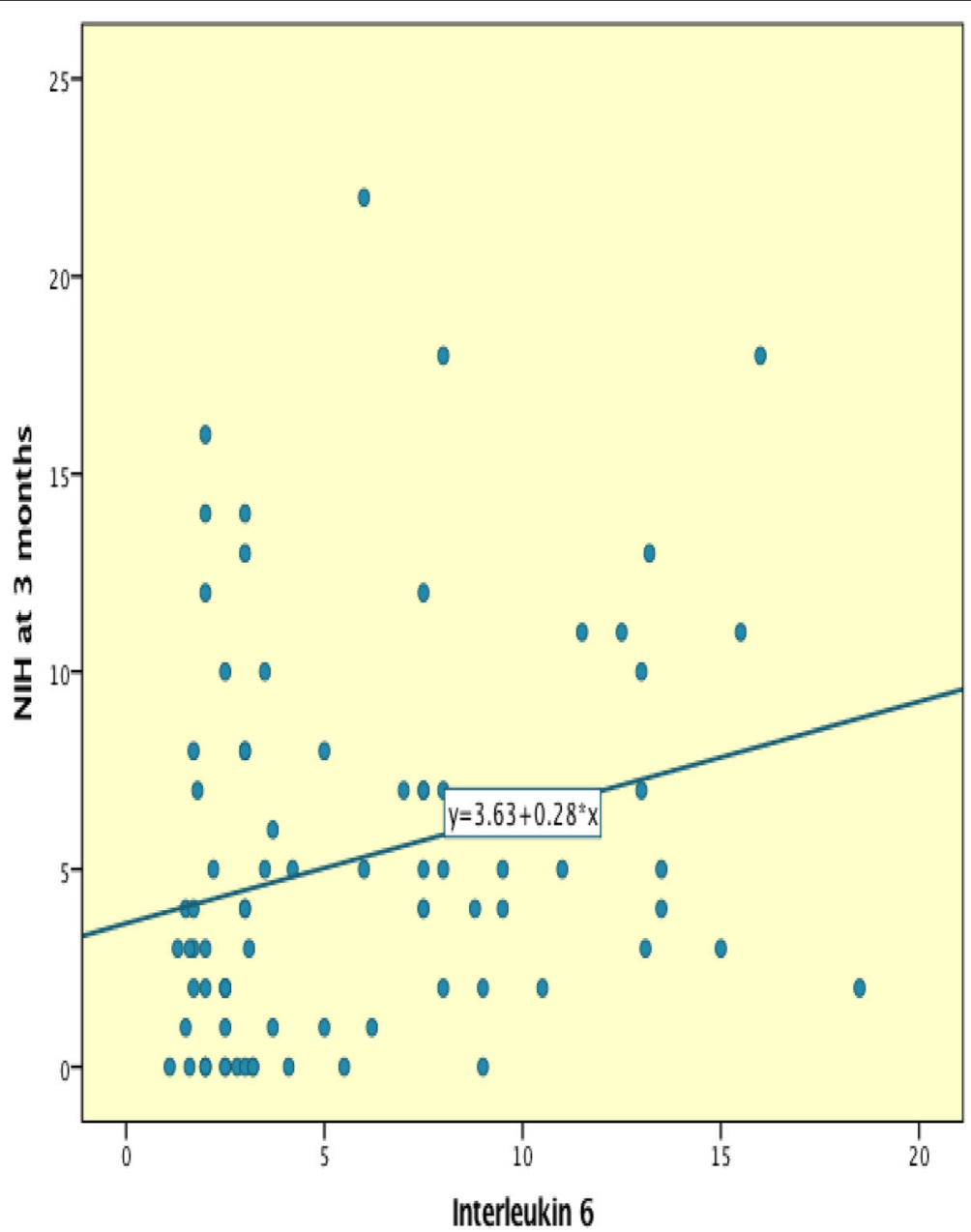

Fig. 1 Graphic representation of the correlation between NIHSS after3 months and IL-6. There was significant positive correlation between IL-6 and NIHSS after 3 months $(P=0.009)(r=0.28)$ 


\section{Compliance, recurrence, and mortality among cases}

Patients were followed up after 3 months to assess their prognosis, compliance on their medications, recurrence of stroke, and mortality (Table 1). Eighty-eight patients (97.7\%) were compliant on their medications, and 15 patients (16.6\%) showed recurrence of the stroke (Table 2). Mortality occurred in 10 patients representing $11.1 \%$ of patients (5 patients due to recurrence, 3 patients due to myocardial infarction, 1 patient due to chest infection, and 1 patient due to dehydration). The mean time to death among those 10 patients was 1.6 months (SD 1.84) (Table 2).

\section{Stroke subtype}

Patients were divided into three groups regarding stroke subtypes (according to TOAST classification) [10]: 38 patients had small vessel occlusion (SVO) (42.22\%), 27 patients had cardioembolic (CE) strokes (30\%), and 25 patients had large artery atherosclerosis (LAA) (27.78\%).

\section{Interleukin- 6 levels in different stroke subgroups}

Overall, the mean value of interleukin-6 was $6.39 \pm$ $4.68 \mathrm{pg} / \mathrm{ml}$. The mean value of IL-6 was $4.64 \mathrm{pg} / \mathrm{ml}$ among patients with cardioembolic stroke, while it was $5.11 \mathrm{pg} / \mathrm{ml}$ among patients with LAA and $8.47 \mathrm{pg} /$ $\mathrm{ml}$ among patients with SVO, denoting statistically significant higher mean value of interleukin-6 in SVO $(P=0.005)$ (Table 3).

\section{Interleukin-6 level and clinical outcome}

There was a non-significant positive correlation between interleukin-6 and NIHSS on admission $(P=0.2) \quad(r=$ $0.135)$, while there was a significant positive correlation between IL-6 and NIHSS after 3 months $(P=0.009)(r=$ 0.28) (Fig. 1 and Table 3).

Also, there was a non-significant positive correlation between interleukin- 6 and $\mathrm{mRS}$ on admission $(P=0.24)$ $(r=0.123)$, while there was a significant positive

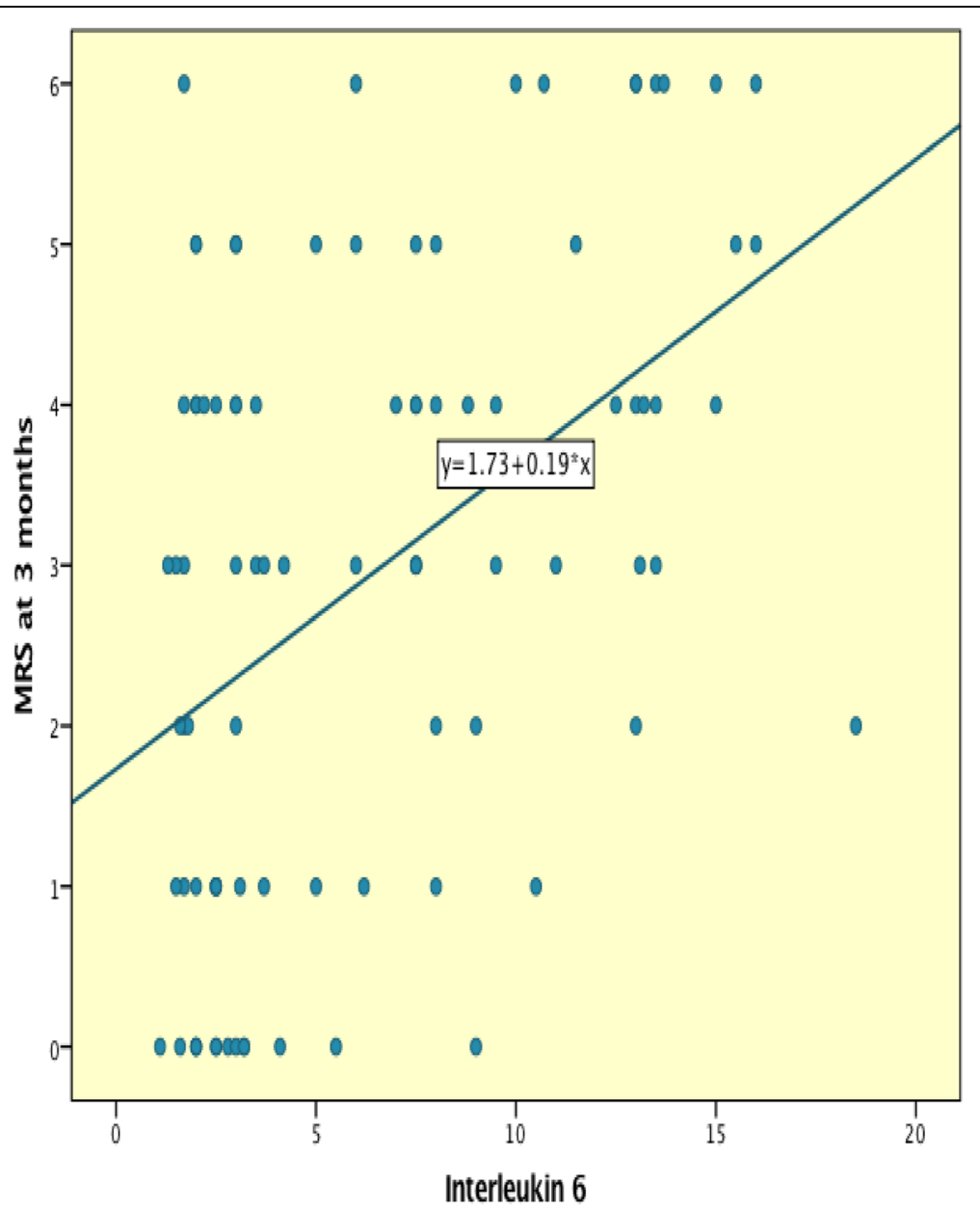

Fig. 2 Graphic representation of the correlation between mRS after 3 months and IL-6. There was a significant positive correlation between IL- 6 and $\mathrm{mRS}$ after 3 months $(P<0.0001)(r=0.42)$ 
correlation between interleukin- 6 and mRS after 3 months $(P<0.0001)(r=0.42)$ (Fig. 2 and Table 3$)$.

\section{Correlation between interleukin- 6 and stroke volume}

A non-significant negative correlation was found between interleukin-6 and stroke volume $(r=-0.213)(P=0.44)$.

\section{Interleukin-6 and recurrence}

Interleukin-6 was significantly higher in patients with recurrence $(12.5 \pm 2.66) \mathrm{pg} / \mathrm{ml}$ than in patients without recurrence $(5.34 \pm 4.11) \mathrm{pg} / \mathrm{ml}(P<0.001)$ (Fig. 3$)$.

\section{Interleukin- 6 and prediction of recurrence}

ROC with AUC analysis was used to test the specificity and sensitivity of interleukin-6 testing to predict recurrence. Area under curve value was 0.9 indicating both strong test specificity and sensitivity in predicting recurrence with a $P$ value of $<0.0001$ (Fig. 4).

The cutoff interleukin- 6 value above which recurrence is anticipated is $7.75 \mathrm{pg} / \mathrm{ml}$ (at which the test sensitivity is 0.93 and the test specificity is 0.75 ).

\section{Discussion}

Different researches of the blood biomarkers associated with cerebral ischemia is ongoing at a rapid pace to define the role of those biomarkers to provide better management of patients with cerebral ischemia [11]. High levels of interleukin-6 in the cerebrospinal fluid (CSF)

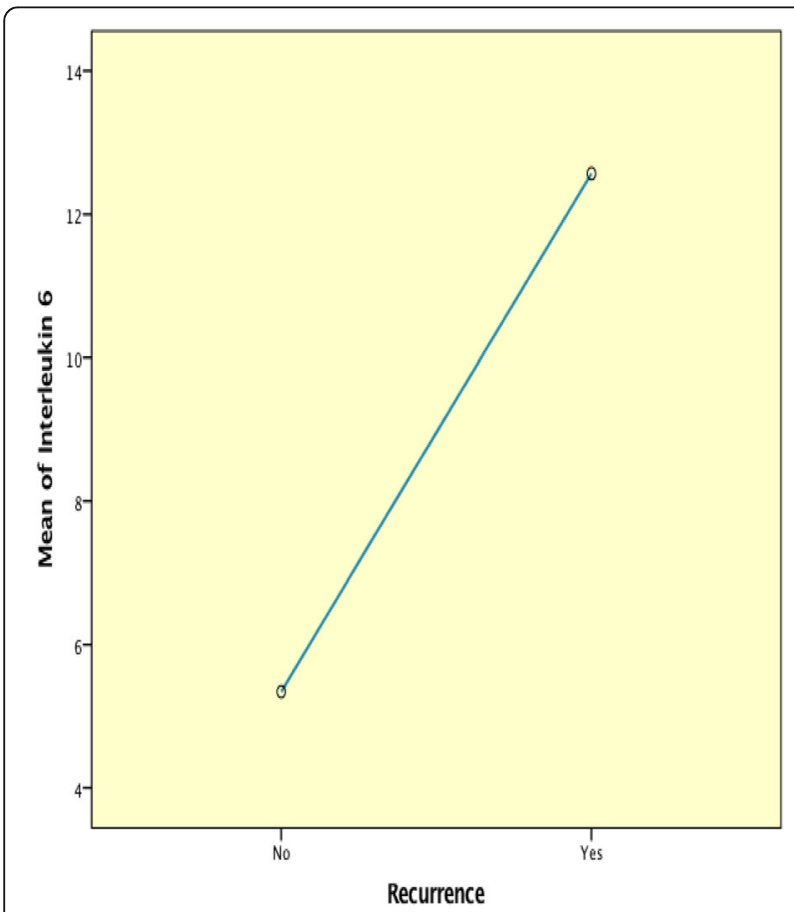

Fig. 3 Means plot between IL-6 levels against recurrence. IL-6 was significantly higher in patients with recurrence $(12.5 \pm 2.66) \mathrm{pg} / \mathrm{ml}$ than in patients without recurrence $(5.34 \pm 4.11) \mathrm{pg} / \mathrm{ml}(P<0.001)$ and blood is associated with early neurological deterioration and poor outcome after cerebral infarction [12].

Our study was set out to assess the role of interleukin6 , as a marker for inflammation in AIS. We studied the level of IL-6 in 90 patients in relation to stroke severity, subtype, and volume and its impact on stroke outcome.

In our study, the average mean value of interleukin- 6 was $6.39 \pm 4.68 \mathrm{pg} / \mathrm{ml}$. Upon comparing means of interleukin-6 values among different stroke subtypes, our study showed statistically significant higher mean value of IL-6 in SVO, followed by LAA then CE strokes. Similar results were described by Hoshi and co-workers [13] who found higher levels of interleukin- 6 in patients with silent brain infarctions suggesting the role of interleukin-6 in SVO.

Infarct size could have direct effect on the inflammatory marker level in blood. Non-significant negative correlation was found between interleukin- 6 and infarct size in our study. Previous studies found conflicting findings regarding interleukin-6 levels and infarct volume. Two studies found no association between serum IL-6 concentration and infarct volume [14, 15], while several studies have shown positive correlations between infarct volume and interleukin- 6 level in serum or plasma $[5$, $11,16]$.

In our study, we assessed the functional status of the studied cases on admission and clinical outcome after 3 months as well as recurrence in correlation with interleukin-6 level. We consider NIHSS more than 21 as poor prognosis and less than 5 as good prognosis. There was non-significant positive correlation between IL-6, mRS, and NIHSS on admission, while there was statistically significant positive correlation between interleukin6, mortality, NIHSS, and mRS after 3 months, denoting worse outcome and higher mortality after 3 months with higher levels of interleukin-6. This means that IL- 6 may have been associated with long-term rather than shortterm outcome. The risk of recurrent stroke is high in the first few weeks after stroke, and clinical risk prediction tools have only limited accuracy [17], hence the importance of identifying a biomarker that can assess recurrence.

In our study on comparing mean values of interleukin-6 among patients with and without recurrence (after exclusion of patients non-compliant on their medications), it was found that there is statistically significant higher mean values of IL-6 in patients with recurrence than mean values of IL-6 in patients without recurrence. It also showed strong test specificity and sensitivity of interleukin- 6 in predicting recurrence. The cutoff IL-6 value above which recurrence is anticipated was $7.75 \mathrm{pg} /$ $\mathrm{ml}$. This demonstrates the clinical potential of using IL-6 as an early predictor for recurrence of acute ischemic stroke and suggests a clear cut point for patients at a high risk who may benefit from closer clinical observation. 


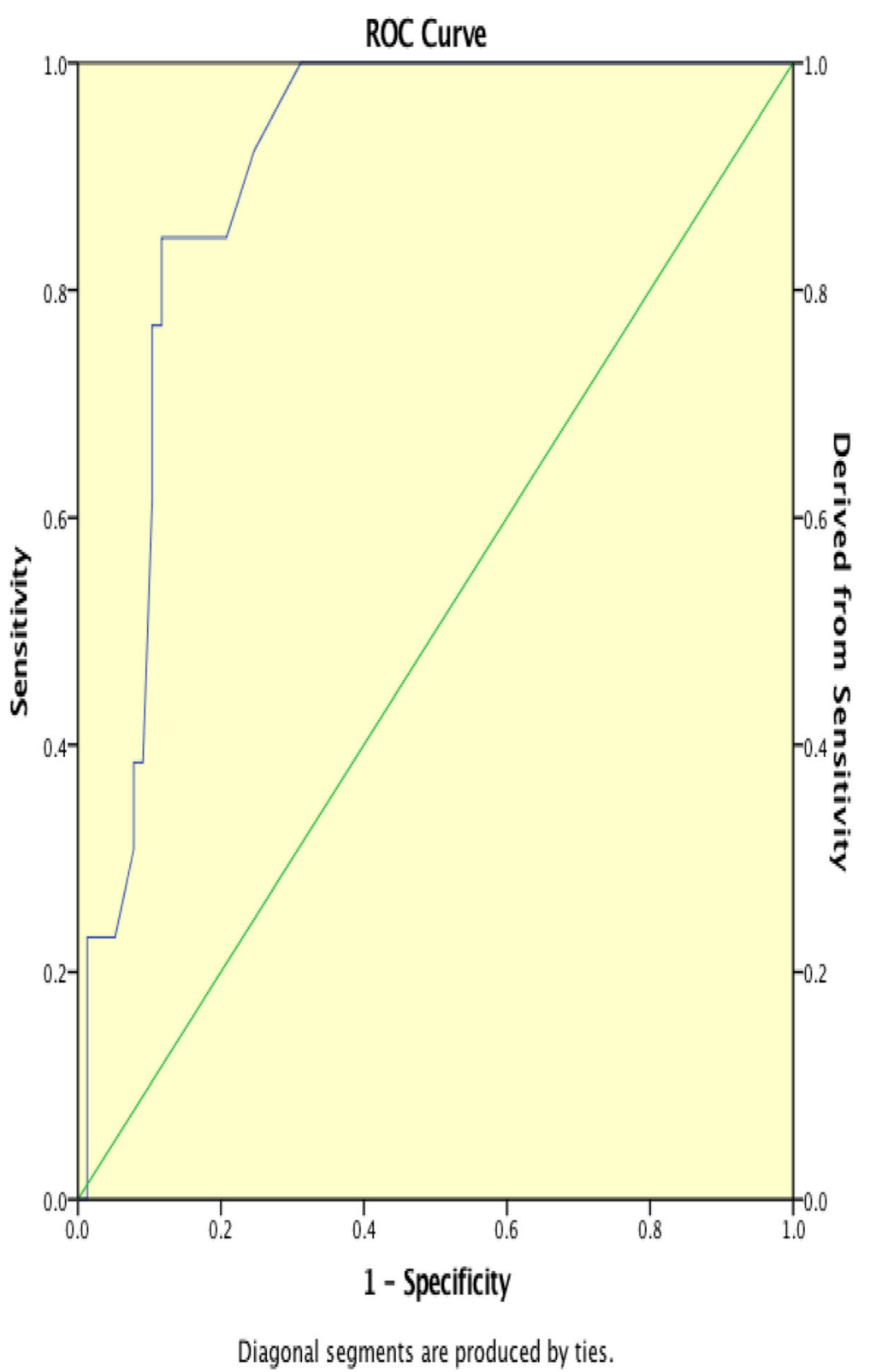

Fig. 4 ROC displaying specificity and sensitivity of IL-6 in predicting recurrence. Receiver operating characteristics (ROC) with area under the curve (AUC) analysis was used to test the specificity and sensitivity of IL-6 testing to predict recurrence. AUC value was 0.9 indicating both strong test specificity and sensitivity in predicting recurrence with a $P$ value of $<0.0001$

A few limitations of our study have to be addressed. First, we did not perform serial measurements of IL-6 in order to select its peak levels. As we assumed that for the clinician in the emergency department, the feasible test will not include serial measurements. A second limitation is that although antiplatelets and statins could have an effect on the number of recurrent strokes, but given the high rate of compliance in our study, we were not able to determine the predictive value of interleukin6 in patients not on medication. Third, we could not use a multifactorial model to assess whether plasma interleukin-6 level was related to outcome independent of risk factors, stroke severity, or infarct size due to the limitation of our sample size.

\section{Conclusion}

Our study concluded that inflammation has an important role in the pathogenesis of acute ischemic stroke particularly small vessel occlusion and it provided evidence that interleukin- 6 contributes to the determination of clinical outcome of AIS and prediction of recurrence, suggesting a role for interleukin- 6 as a prognostic marker. 


\section{Recommendations}

Interleukin- 6 could be applied in the routine clinical settings as an additive prognostic tool (together with clinical features, radiological investigations, and risk factors) in patients with AIS. This important marker should be used as a predictor of stroke recurrence, for the early identification and monitoring of patients at high risk of recurrence.

\section{Abbreviations}

AUC: Area under the curve; CE: Cardioembolic; CRP: C-reactive protein; CSF: Cerebrospinal fluid; ELISA: Enzyme-linked immunosorbent assay; IL6: Interleukin-6; LAA: Large artery atherosclerosis; MCA: Middle cerebral artery; MRA: Magnetic resonance angiography; MRI: Magnetic resonance imaging: mRS: Modified Rankin Scale; NIHSS: National Institute of Health and Stroke Scale; ROC: Receiver operating characteristics; TOAST: Trial of ORG 10712 in Acute Stroke Treatment

\section{Acknowledgements}

Not applicable

\section{Authors' contributions}

HMAA, NAF, AE, and MOA conceived of the study and participated in its design and coordination and helped to draft the manuscript (FG). SHK participated in the sequence alignment (MT). MFA participated in the design of the study and performed the statistical analysis (ES). All authors read and approved the final manuscript.

\section{Funding}

Not applicable

\section{Availability of data and materials}

Dataset is available as master sheet in Excel format and publicly available in the Neurology Department, Ain Shams University.

\section{Ethics approval and consent to participate}

The study was approved by Ain Shams University Ethical Committee in December 2014. Written informed consent was obtained from the patients participating in the study, or their first-degree relatives if the patient was unable to provide consent due to weakness or disturbed conscious level, after informing them about the study rationale and their right to withdraw from the study at any time without any consequences.

\section{Consent for publication}

Not applicable

\section{Competing interests}

The authors declare that they have no competing interests (regarding

financial and non-financial competing interests).

Received: 7 August 2018 Accepted: 6 October 2019

Published online: 16 January 2020

\section{References}

1. Muir K, Tyrrell P, Sattar N, Warburton E. Inflammation and ischaemic stroke. Curr Opin Neurol. 2007;20(3):334-42.

2. Lambertsen K, Biber K, Finsen B. Inflammatory cytokines in experimental and human stroke. J Cereb Blood Flow Metab. 2012;32(9):1677-98.

3. Tuttolomondo A, Di Raimondo D, Pecoraro R, Arnao V, Pinto A, Licata G. Inflammation in ischemic stroke subtypes. Curr Pharm Des. 2012; 1(18):4289-310.

4. Andreassen U, Kråkenes J, Ulvestad E, Thomassen L, Myhr KM, Aarseth J, Vedeler CA. IL-6: an early marker for outcome in acute ischemic stroke. Acta Neurol Scand. 2005:111(6):360-5.

5. Shaafi S, Sharifipour E, Rahmanifar R, Hejazi S, Andalib S, Nikanfar M, Baradarn B, Mehdizadeh R. Interleukin-6, a reliable prognostic factor for ischemic stroke. Iran J Neurol. 2014;13(2):70-6.

6. Herve D, Mangin JF, Molko N, Bousser MG, Chabriat H. Shape and volume of lacunar infarcts: a 3D MRI study in cerebral autosomal dominant arteriopathy with subcortical infarcts and leukoencephalopathy. Stroke. 2005;36:2384-8.

7. Ridker P, Rifai N, Stampfer M, Hennekens CH. Plasma concentration of interleukin- 6 and the risk of future myocardial infarction among apparently healthy men. Circulation. 2000;101(15):1767-72.

8. Yamamura M, Yamada Y, Momita S. Circulating interleukin-6 levels are elevated in adult T-cell leukaemia/lymphoma patients and correlate with adverse clinical features and survival. Br J Haematol. 1998;100:129-34.

9. Easton J, Saver J, Albers G, Alberts MJ, Chaturvedi S, Feldmann E, et al. Definition and evaluation of transient ischemic attack: a scientific statement for healthcare professionals from the American Heart Association/American Stroke Association Stroke Council; Council on Cardiovascular Surgery and Anesthesia; Council on Cardiovascular Radiology and Intervention; Council on Cardiovascular Nursing; and the Interdisciplinary Council on Peripheral Vascular Disease. Stroke. 2009;40(6):2276-93.

10. Adams H, Bendixen B, Kappelle $L$, et al. Classification of subtype of acute ischemic stroke. Definitions for use in a multicenter clinical trial. TOAST. Trial of Org 10172 in Acute Stroke Treatment. Stroke. 1993;24:35-41.

11. Acalovschi D, Wiest T, Hartmann M, Farahmi M, Mansmann U, Auffarth GU, Grau AJ, Green FR, Grond-Ginsbach C, Schwaninger M. Multiple levels of regulation of the interleukin-6 system in stroke. Stroke. 2003;34(8):1864-9.

12. Rodriguez-Yanez M, Castillo J. Inflammation in brain ischemia, the role of markers. Curr Opin Neurol. 2008;21(3):353-7.

13. Hoshi T, Kitagawa K, Yamagami H, Furukado S, Hougaku H, Hori M. Relations of serum high-sensitivity C-reactive protein and interleukin-6 levels with silent brain infarction. Stroke. 2005;36(4):768-72.

14. Ormstad H, Aass H, Lund-Sorensen N, Amthor KF, Sandvik L. Serum levels of cytokines and C-reactive protein in acute ischemic stroke patients, and their relationship to stroke lateralization, type, and infarct volume. J Neurol. 2001; 258(4):677-85.

15. Sahan M, Sebe A, Acikalin A, Akpinar O, Koc F, Ay MO, Gulen M, Topal M, Satar S. Acute-phase reactants and cytokines in ischemic stroke: do they have any relationship with short-term mortality? Eur Rev Med PharmacolSci. 2013;17(20):2773-7.

16. Vila N, Castillo J, Dávalos A, Chamorro A. Proinflammatory cytokines and early neurological worsening in ischemic stroke. Stroke. 2000;31(10):2325-9.

17. Mohan K, Wolfe C, Rudd A, Heuschmann P, Kolominsky-Rabas PL, Grieve A. Risk and cumulative risk of stroke recurrence: a systematic review and metaanalysis. Stroke. 2011;42(5):1489-94.

\section{Publisher's Note}

Springer Nature remains neutral with regard to jurisdictional claims in published maps and institutional affiliations.

\section{Submit your manuscript to a SpringerOpen ${ }^{\circ}$ journal and benefit from:}

- Convenient online submission

- Rigorous peer review

- Open access: articles freely available online

High visibility within the field

- Retaining the copyright to your article

Submit your next manuscript at $>$ springeropen.com 